\title{
LATTICE DESIGN OF A CARBON-ION SYNCHROTRON FOR CANCER THERAPY*
}

\author{
H. S. Kang ${ }^{\dagger}$, H. S. Suh \\ Pohang Accelerator Laboratory, POSTECH, Pohang, Kyungbuk, 790-784 KOREA
}

\begin{abstract}
A medical synchrotron for carbon-ion cancer therapy was designed to be compact for a hospital-based therapy facility. The circumference of the synchrotron is only 60 meter, and the lattice is a FODO structure of 6 cells. The lattice satisfies the requirements for resonant slowbeam extraction. An acceleration-driven resonant extraction scheme using betatron core is chosen to offer the smoothest spill of particles to the patients. Particle tracking simulations for injection and extraction are carried out to confirm the lattice design.
\end{abstract}

\section{INTRODUCTION}

Cancer therapy using carbon ions has been highlighted for the past ten years worldwide because of its superior characteristics, such as high RBE (relative biological effectiveness) and small beam diffraction. Carbon-ion cancer therapy facilities are being constructed in Europe and Japan $[1,2]$ and were activated after a design study for a medical synchrotron. The design study was carried out within the framework of the Proton Ion Medical Machine Study (PIMMS) [3]. The typical design of a carbon-ion cancer therapy facility is to provide not only a carbon ion beam by high precision active scanning (raster scanning) but also a proton beam by passive spreading. It also should provide smooth spill and reliability and simplicity of operation.

The quality of the dose intensity profile in the patient's body ultimately depends on the uniformity of the beam spill from the synchrotron. In order to get a dose uniformity of less than $\pm 2.5 \%$, the medical synchrotron requires a very stable extraction of the beam over a period of about one second. Also, the beam scanning treatment system should monitor the particle flux entering the patient with an on-line measurement system so that the beam spill can be extended in time (seconds).

If the uniformity requirements of the spill are to be satisfied, the momentum spread of the extracted beam should be small, and the mean energy and the spread must not vary within a single spill. Thus, the most stringent requirement comes from the beam extraction. The synchrotron lattice needs to satisfy the requirement of the Hardt condition for slow beam extraction, which is to align the separatrices of different momenta of the particles [4], thereby making the extraction angle independent of the particle momentum error.

\footnotetext{
${ }^{*}$ Work supported by Korean Ministry of Science and Technology

${ }^{\dagger}$ hskang@ postech.ac.kr
}

The typical design satisfying these requirements, for example, the PIMMS design, has a circumference of 80 meter, which is too big to be used for a hospital-based facility. The uniformity of the spill during extraction depends on how well the lattice and the extraction scheme are designed, which are obviously idealized in the PIMMS design. However, the lattice needs to be optimized further for reducing the circumference because there are many choices in the lattice design. The PIMMS design is just one choice. In this regard a synchrotron accelerator for carbon ion cancer therapy was designed to be compact by optimizing the lattice itself without losing the concepts proposed in the PIMMS design.

\section{REQUIREMENTS OF SYNCHROTRON}

The accelerator requirements for a carbon-ion cancer therapy facility are as follows: 1) a penetration depth of $38 \mathrm{~cm}$ in water, which is available at $250 \mathrm{MeV}$ for protons and $430 \mathrm{MeV} / \mathrm{u}$ for carbon, and (2) a daily dose of typically around 2 Gray $(\mathrm{J} / \mathrm{kg})$ delivered in 1 or 2 minutes, which requires a fast cycle of operation. The beam intensity of the carbon ion is $4 \times 10^{9} \mathrm{ppp}$ (particles per pulse). The facility is comprised of an ion source, which generates a proton or a carbon-ion beam, a RFQ (radio-frequency quadrupole), a IH-linac, a synchrotron, and a transfer line to the gantry. The carbon ion from the ion source is ${ }^{12} C^{+4}$, which is accelerated in the RFQ and the IH-linac to the injection energy and is then charge-striped to ${ }^{12} C^{+6}$ before injection to synchrotron. In the synchrotron, the beam is to be trapped, accelerated, rebunched to change harmonics, followed by a stabilized start of spill and extraction. In the synchrotron, there should be dispersion-free straight sections in which are located the septum magnets for injection and extraction, a resonance sextupole, and an RF cavity.

The slow beam extraction method from the synchrotron is a third-integer resonant scheme driven by sextupoles. The third-integer, resonant extraction system uses the simplified Kobayashi hamiltonian, $H$, that describes the particle motion perturbed by the resonance sextupole in the plane of extraction:

$$
H=\frac{\epsilon}{2}\left(X^{2}+X^{\prime 2}\right)+\frac{S_{n}}{4}\left(3 X X^{\prime 2}-X^{3}\right),
$$

where $\epsilon=6 \pi \Delta \nu, \Delta \nu$ being the distance from the resonance $\nu=n \pm 1 / 3$, and $S_{n}$ is the normalized resonance sextupole strength defined by

$$
S_{n}=\frac{1}{2} \beta_{x, \text { sext }}{ }^{3 / 2} S l_{\text {sextupole }},
$$




$$
S=\frac{\partial^{2} B_{z}}{\partial x^{2}} \frac{1}{B \rho},
$$

with $\beta_{x, \text { sext }}$ being the horizontal beta function at the resonance sextupole, $l_{\text {sextupole }}$ the length of the sextupole, and $B \rho$ the magnetic rigidity of the particle beam. $S_{n}$ is controlled by the resonance sextupole, which should be located in a dispersion-free section so as not to introduce additional chromaticity.

The techniques for applying resonant extraction to achieve a long spill (seconds) can be classified into two groups, those that move the resonance (quadrupole-driven extraction) and those that move the beam (accelerationdriven extraction, RF knockout extraction). The latter has the advantage of keeping all lattice functions, hence, the resonance conditions constant.

The acceleration-driven extraction scheme moves the beam into a stationary resonance by accelerating the beam. It doesn't need to change the tune so that the transverse optics of the ring can be kept constant through the extraction, which means there is no movement of the orbit and the extraction separatrices. It can offer smooth spill in the extraction, which is why this method is preferred for slow extraction. The beam acceleration can be done by using a betatron core or a stochastic noise system.

In this synchrotron lattice design, the acceleration-driven extraction scheme using a betatron core is chosen because it offers the smoothest spill of particles. As an alternative, a quadrupole-driven extraction scheme will also be prepared because it does not need any additional equipment.

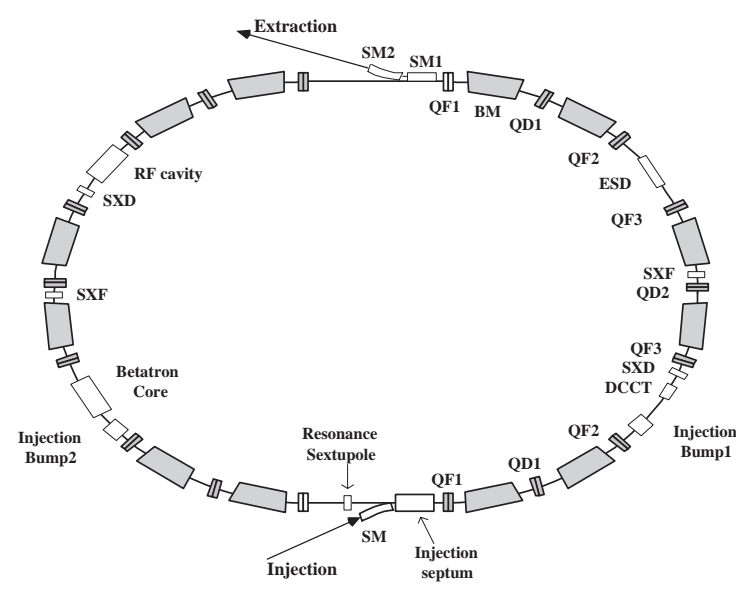

Figure 1: Layout of the synchrotron (BM: dipole bending magnet, QF: quadrupole focusing, QD: quadrupole defocusing, ESD: electrostatic deflector, and SX: sextupole.

\section{LATTICE DESIGN}

The lattice design was done with MAD and WinAgile $[5,6]$. Figure 1 depicts the schematic layout of the designed synchrotron. The circumference of the synchrotron is only 60 meter, and the lattice is a FODO structure of 6 cells. Each cell has two dipole magnets with a bend angle of $30^{\circ}$. The lattice has two superperiods. Each superperiod consists of 3 FODOs with a symmetry at the mid-point. One resonance sextupole is located in the dispersion-free section and two chromaticity sextupoles of equal strength are positioned in the dispersion regions of the arcs on opposite sides of the ring where the resonance driving term is zero. The horizontal chromaticity sextupoles (SXF) are located at a large $\beta_{x}$ and small $\beta_{z}$ (vertical betafunction), and the converse (SXD) applies to the vertical ones. In this layout, independent chromaticity and resonance control is possible. For injection, a septum magnet (SM) and an electrostatic septum (injection septum) and two bump magnets (injection bump1, injection bump2) will be used. For extraction, a resonance sextupole, a betatron core, an ESD and two septum magnets (SM1, SM2) will be used.

Figure 2 shows the Twiss parameters of the whole lattice. The two straight sections for the injection and the extraction septum have zero dispersion. The magnetic rigidity of the $430-\mathrm{MeV} / \mathrm{u}$ carbon ion is $6.64 \mathrm{~T}-\mathrm{m}$. The tune $\left(\nu_{x}, \nu_{z}\right)$ is $1.68,1.13$ and $\Delta \nu=0.0133$. The injection energy of the carbon ion is $4 \mathrm{MeV} / \mathrm{u}$, and the magnetic rigidity is 0.578 $\mathrm{T}-\mathrm{m}$. The operating cycle for the carbon ion has a $0.5-\mathrm{sec}$ ramp, a 1.0-sec spill, and a 0.5-sec de-ramp. The extraction method is to use betatron core, and a quadrupole-driven scheme will be prepared as an alternative.

The lattice satisfies the Hardt condition for resonant beam extraction at the location of the electrostatic deflector where $D=4.97, D^{\prime}=-0.938, \beta_{x}=11.1$, and $\alpha_{x}=0.594$. Therefore, $D_{n}=\frac{D}{\sqrt{\beta_{x}}}=1.49$ and $D_{n}{ }^{\prime}=\sqrt{\beta_{x}} D^{\prime}+\left(\alpha_{x} / \sqrt{\beta_{x}}\right) D=-2.239$. The betatron phase difference between the ESD and the septum magnet SM1 is $68^{\circ}$, which is close to $90^{\circ}$, enough for the extraction at the following septum (SM1), and the betatron phase difference between the resonance sextupole and the ESD is $227^{\circ}$. The resonance sextupole strength $S_{n}$ and the chromaticity can be adjusted to satisfy the Hardt condition. The relationship between $S_{n}$ and $\xi_{x}$ is $\left|\xi_{x}\right|=0.207 \times S_{n}$. For $S_{n}=18.5(S=5)$, the horizontal chromaticity needs to be set at -3.7 .

The betatron core will be used to accelerate a beam of small emittance and large momentum spread into the resonance. The application of a time-varying current $I(t)$ to the betatron coil excites a magnetic flux variation $\Delta \phi$, which produces an electric field parallel to the axis of the betatron. The accelerating field that pushes the beam into the resonance is largely independent of the beam characteristics, thus giving a uniform acceleration. The necessary magnetic flux variation inside the iron core of the betatron required for a given momentum change $(\Delta p)$ is

$$
\Delta \phi=C B \rho \frac{\Delta p}{p}
$$

where $C$ is the circumference, $B \rho$ is the magnetic rigidity [7].

The total momentum variation required to extract all the particles in the acceleration-driven extraction scheme is 

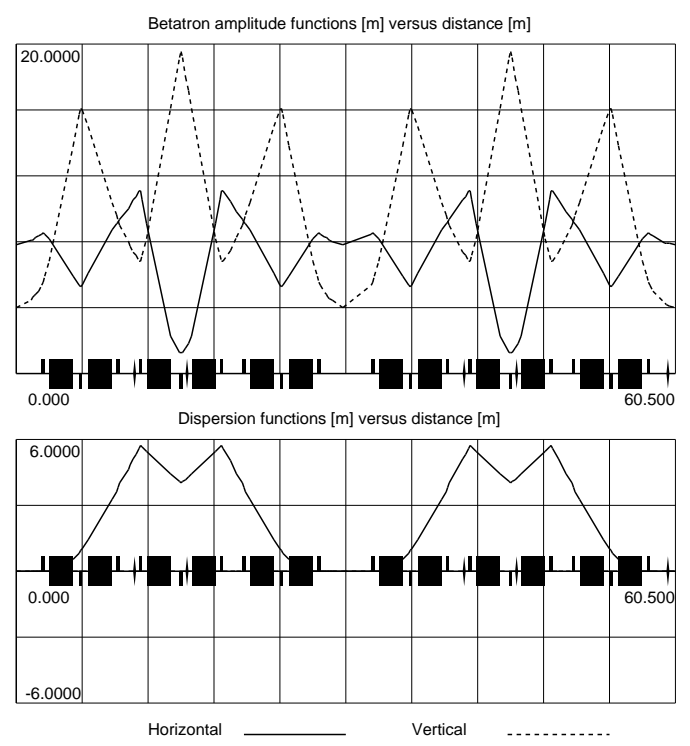

Figure 2: Twiss parameters of the lattice (tune: 1.68/1.13). Betatron amplitude function vs. distance (upper figure) and dispersion function vs. distance (lower)

$\Delta p / p=0.5 \%$. It is assumed that the extraction process starts with a stack positioned at a $\Delta p / p=0.1 \%$ from the resonance and that the stack has up to $\Delta p / p=0.4 \%$ due to the betatron core. From Eq. (4), the total magnetic flux variation needed in the betatron to sweep the whole stack through the resonance is $\Delta \phi=1.59 \mathrm{~Wb}$. In order to reduce the size of the core, the betatron core is designed to generate a half of the required magnetic flux variation of $\Delta \phi=1.59 \mathrm{~Wb}$ so that two betatron cores need to be prepared.

Particle-tracking simulations for injection and extraction were carried out by using the code WinAgile. A beam extraction simulation was done for two schemes: a quadrupole-driven extraction and an acceleration-driven extraction. Figure 3 shows the horizontal and the vertical phase spaces of carbon-ion beam at the position of the ESD after 25 turns in the acceleration-driven extraction scheme. The transverse tune is set to $1.68 / 1.13$, and $\Delta \nu$, the distance from the resonance, is 0.0133 . The momentum spread obtained by acceleration using the betatron core is assumed to be (a) $0.3 \%$ and (b) $0.5 \%$. The resonance sextupole strength is set to $5\left(S_{n}=18.5\right)$. The separatrice for extraction is located in the fourth plane $((+)$ on the horizontal axis and (-) on the vertical axis) of the horizontal phase space in Fig. 3. The extraction angles for both $0.3 \%$ and $0.5 \%$ are around $-3 \mathrm{mrad}$, which means the extraction angle is independent of the momentum error and that the lattice satisfies the Hardt condition for resonant beam extraction.

\section{SUMMARY}

A medical synchrotron for carbon ion cancer therapy was designed to be compact for a hospital-based therapy facility. The lattice satisfies the Hardt condition for reso-
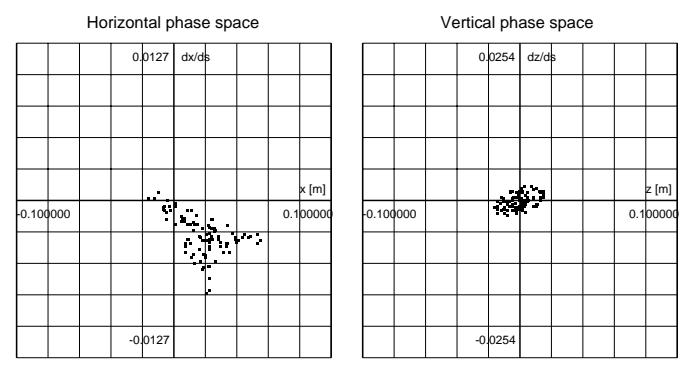

(a)
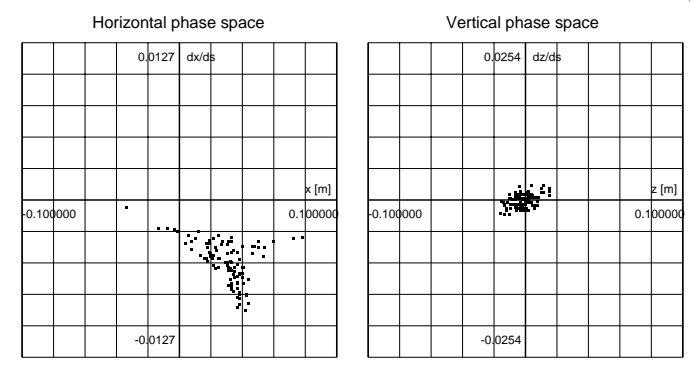

(b)

Figure 3: Horizontal and vertical phase spaces of a carbon ion beam after 25 turns in the acceleration-driven extraction scheme. The momentum spread is assumed to be (a) $0.3 \%$ and (b) $0.5 \%$. The carbon beam energy is $400 \mathrm{MeV} / \mathrm{u}$, and the resonance sextupole strength is set to 5 .

nant beam extraction. The acceleration-driven resonant extraction scheme using a betatron core is chosen to offer the smoothest spill of the particles to the patients. The particletracking simulation for injection confirms the required conditions of momentum acceptance and injection energy for the carbon-ion beam. The particle-tracking simulation for extraction shows that the designed lattice satisfies the Hardt condition to align the separatrices of different momenta of the particles and, therefore, to make the extraction angle independent of the momentum error.

\section{REFERENCES}

[1] Sandro Rossi, in Proceedings of the European Particle Accelerator Conference 2006, Edinburgh, Scotland, p. 3631.

[2] Jean-Michel Lagniel, in Proceedings of the Particle Accelerator Conference 2007, Albuquerque, New Mexico, p. 127.

[3] Accelerator Comples Study Group, "Proton-ion medical machine study (PIMMS) Part I," CERN/PS 99-010, 1999.

[4] L. Badano et al., Nucl. Instr. Meth. A430, 512 (1999).

[5] H. Grote and F. Iselin, The MAD program, Users' Reference Manual, CERN/SL/90-13 (AP), 1990.

[6] P. J. Brynt, in Proceedings of the European Particle Accelerator Conference 2000, Geneva, Switzerland, p. 1357.

[7] P. Knaus et al., P. A. Chamouard, in Proceedings of the European Particle Accelerator Conference 1998, p. 2368. 